\title{
Effect of a Gel Retainment Dam on Automated Ultrasound Coverage in a Dual-Modality Breast Imaging System
}

\author{
Jie Li, MD, Mitchell M. Goodsitt, PhD, Frederic Padilla, PhD, J. Brian Fowlkes, PhD, \\ Fong Ming Hooi, MS, Christine R. Lashbrook, RT(R)(M), Kai E. Thomenius, PhD, \\ Paul L. Carson, PhD
}

\begin{abstract}
Abbreviations
$A B U$, automated breast ultrasound; DBT, digital breast tomosynthesis; 3D, 3-dimensional
\end{abstract}

Received November 16, 2009, from the Department of Radiology, University of Michigan Health Systems, Ann Arbor, Michigan USA (J.L., M.M.G., F.P., J.B.F., F.M.H., C.R.L., P.L.C.); Department of Ultrasound, Qilu Hospital, Shandong University, Jinan, China (J.L.); Laboratoire d'Imagerie Paramétrique, Centre National de la Recherche Scientifique, Unité Mixte de Recherche 7623, Université Pierre et Marie Curie, Paris, France (F.P.); and GE Global Research, Niskayuna, New York USA (K.E.T.). Revision requested December 10, 2009. Revised manuscript accepted for publication March 4, 2010.

This work was supported in part by US Public Health Service grant R01 CA91713. Others contributing recently to the combined system used in this project were Andrea Schmitz, MS (GE Global Research), and Gerald L. LeCarpentier, PhD, Ganesh Narayanasamy, MS, Sumedha P. Sinha MS, and Marilyn Roubidoux, MD (Department of Radiology, University of Michigan Health Systems). Dr Thomenius is an employee of GE Global Research.

Address correspondence to Paul L. Carson, PhD, Department of Radiology, University of Michigan Health Systems, 3218C Medical Science I, B Wing, SPC 5667, 1301 Catherine St, Ann Arbor, MI 481095667 USA.

E-mail: pcarson@umich.edu
Objective. The goal of this work was to evaluate a possible improvement in ultrasound coverage for a dual-modality breast imaging system in the mammographic geometry. Methods. A pilot study was performed to evaluate use of a rubber dam to retain ultrasound gel and improve imaging coverage at the breast periphery on a combined imaging system consisting of an ultrasound scanner and a digital x-ray tomosynthesis unit. Several dams were constructed to encompass the shapes of various sizes of compressed breasts. Visual tracings of the breast-to-paddle contact area and breast periphery were made for 8 breasts to estimate coverage area. Two readers independently reviewed the resulting images and were asked to rate the overall breast image quality. Results. The percentages of breast in contact with the paddle were greater $(P<.01)$ and the linear dimensions of breast in contact with the paddle were larger $(P<.05)$ with the rubber dam than without it. With the dam, the mean estimated area of the breast in contact with the paddle increased $14 \%$, whereas the mean increase in the fraction of the total breast area in contact with paddle was $30 \%$. The difference was due to the mean total projected area of the breast decreasing $12 \%$ as the dam was pressed against it. The image quality of automated ultrasound with the rubber dam was consistently judged to be superior to that without the dam. Conclusions. This method can enhance the absolute and percentage area of the breast in contact with the paddle, reducing noncontact gaps at the breast periphery. Gently pressing the breast periphery with the dam inserted toward the chest wall improves coverage in automated breast ultrasound scanning. Key words: automated ultrasound; breast cancer screening; multimodality; 3-dimensional imaging.

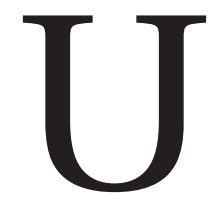

ltrasound imaging has been found to be a valuable adjunct to mammography in the characterization of breast lesions. ${ }^{1,2}$ However, conventional ultrasound imaging is performed freehand in a different geometry than mammography, which can make it difficult to correlate lesions between the two modalities. Studies have shown that at least $10 \%$ of the time, lesions found in the ultrasound images do not correspond with those in the mammograms. ${ }^{3}$ False-positive results are a particular problem when searching for secondary masses and for detection in high-risk or screening populations. ${ }^{4,5}$ 
A combined x-ray/ultrasound system addresses these problems by first taking a 3-dimensional (3D) x-ray image (tomosynthesis) of a compressed breast and then scanning a highfrequency (9- to 12-MHz) ultrasonic transducer across a special dual-modality compression paddle while the breast is still under compression. This creates co-registered 3D x-ray and 3D ultrasound images in the same imaging geometry. ${ }^{6,7}$ Dual-mode whole-breast imaging also has considerable potential for advanced $\mathrm{x}$-ray and ultrasound modes, which would provide additional information about breast tissues that is not available from conventional ultrasound and mammography. For example, in its current configuration, x-ray digital breast tomosynthesis (DBT) replaces single-projection digital mammography in the combined system for 3D delineation of tissue structures and better correlation with 3D ultrasound. ${ }^{8}$ Initial results of the fusion of pulse-echo automated breast ultrasound $(\mathrm{ABU})$ and DBT are promising, although there remain technical issues of breast coverage and some acoustic coupling artifacts in ABU., ${ }^{8,9}$

A major source of the coverage problems in performing automated ultrasound scans in a dualmodality system is that there can be an appreciable air gap between the compression paddle and the breast surface near the breast periphery. ${ }^{8,10,11}$ In our previous work, we used a viscous gel that was manually distributed with a syringe at the breast periphery to address the air gap problem. ${ }^{8}$ In most of our patient studies, where needed, we also taped a thin strip of plastic film around the breast periphery to act as a dam to keep the viscous gel in place. However, the effects of these dams were not quantified or reported. The dams used in this study were made of butyl rubber to provide an absorptive, minimally reflecting surface to minimize artifactual reflections.

\section{Materials and Methods}

This study was approved by the Institutional Review Board, and informed consent was obtained from all participants. Trials were performed on a combined system consisting of a LOGIQ 9 ultrasound system (GE Healthcare, Milwaukee, WI) and a second-generation GE/University of Michigan research DBT unit (Figure 1). A linear matrix array ultrasound transducer (GE M12L) operating at a center frequency of $12 \mathrm{MHz}$ was translated across a compression paddle with a motorized transducer carriage. This ABU scanning system was developed jointly with GE.

The participant was seated comfortably throughout the scan to minimize motion artifacts and fatigue, and the breast was positioned between the compression paddle and digital $\mathrm{x}$ ray detector. Instead of the solid polymethylpentene compression paddle described previously, ${ }^{6-9}$ a fiber mesh paddle similar to a fine mesh tennis racket was used in this study. ${ }^{12}$ This new paddle, similar to but with a finer mesh than one recently described, makes acoustic coupling easier and better between the transducer and the breast surface. An evaluation of various mesh paddle

Figure 1. A, Dual-modality combined system consisting of a GE LOGIQ 9 ultrasound system and a second-generation GE digital tomosynthesis unit. B, Breast-simulating phantom illustrating an air gap between the phantom and the compression paddle at the periphery.

A

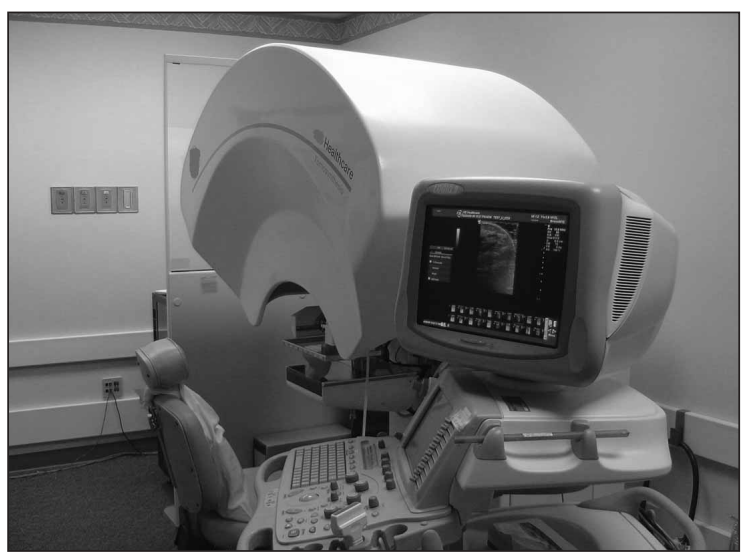

B

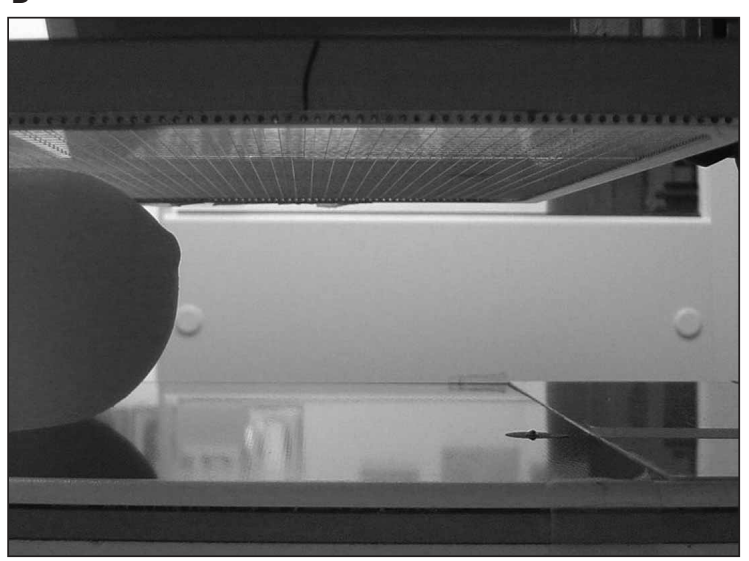


designs for our dual-modality system will be presented in the near future. A compression force of approximately 4 to $8 \mathrm{dN}$ was typically used with the dual-modality system to minimize patient

Figure 2. A and B, Rubber dams constructed by adhering two 0.2 -cm-thick plastic sheets to a strip of 0.15 -cm-thick butyl rubber and a rubber hinge. $\mathbf{C}$, The inside shape adequately mimics that of the breast in this lateral view as well as other views.

A

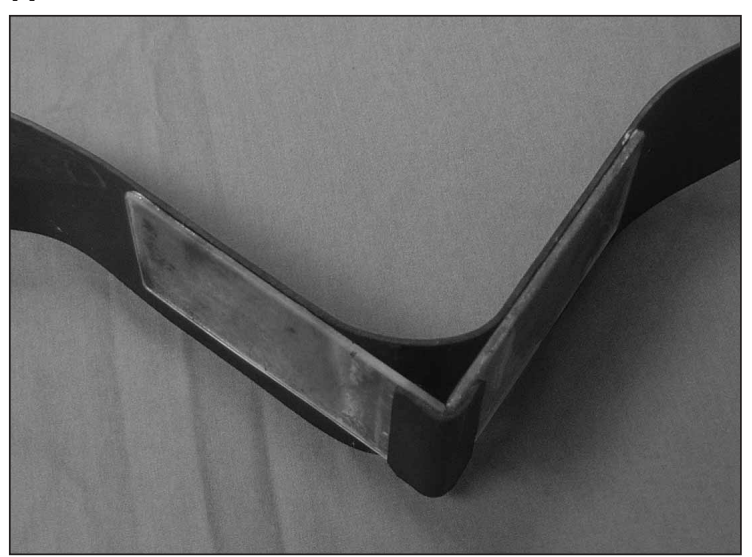

B

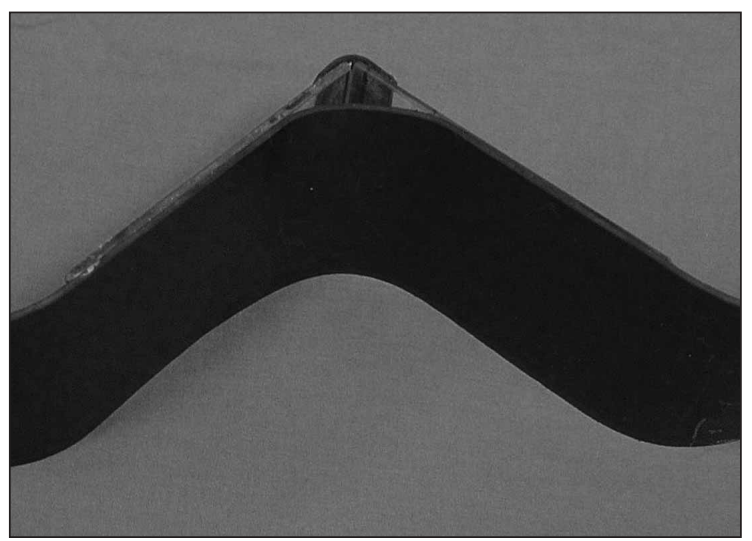

c

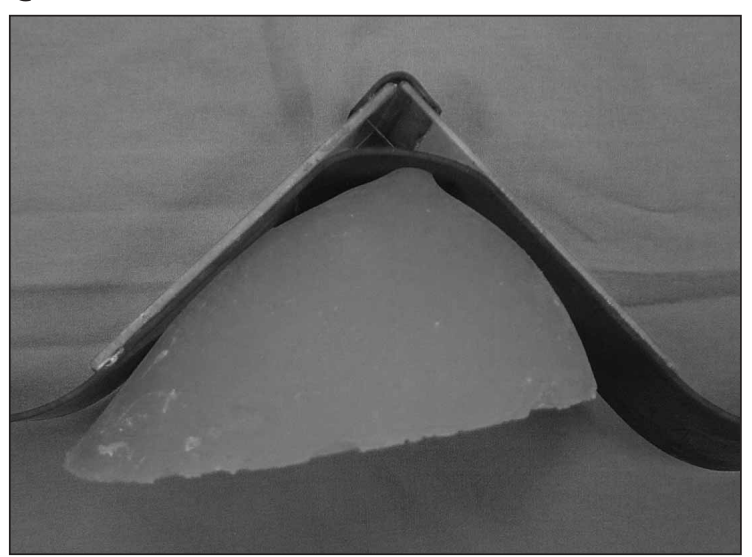

discomfort while stabilizing the breast. Eight breasts of 4 healthy female volunteers were imaged with this combined system. The ages of the 4 healthy volunteers ranged from 45 to 54 years (mean, 51 years). Two automated scans of each breast were performed. One scan was conducted without using the rubber dam. For the second scan, the rubber dam was deployed around the breast periphery and pushed a little toward the chest wall. Bubble-free ultrasound gel was used as the coupling medium between the transducer and the breast surface through the fiber mesh compression paddle for craniocaudal views.

Rubber dams were constructed by adhering two $0.2-\mathrm{cm}$-thick plastic sheets to a strip of 0.15$\mathrm{cm}$-thick butyl rubber. The dams were made to mimic the shape of the breast (Figure 2). Rubber is advantageous because it can reduce artifacts by absorbing ultrasound reflections but is too soft to use by itself. Plastic, on the other hand, is hard and maintains its structure but produces more artifacts because of the relatively strong reflections at its interfaces with water and air, its support of shear waves, and its low attenuation coefficient relative to rubber. The combination of rubber supported by a plastic backing maintains the advantages of both materials. Six dams were constructed to accommodate an angle range from $30^{\circ}$ to $120^{\circ}$ with heights of $2.0,2.5$, $3.5,4.0,5.0$, and $6.0 \mathrm{~cm}$. The variable angle of the $\mathrm{V}$ shape and height differences of these dams accommodate the different breast sizes of patients.

To estimate the fraction of the breast surface area that was in contact with the compression paddle, we used a method described previously. ${ }^{8}$ In brief, thin transparency film was placed on the compression paddle. Using a marking pen, the technologist made visual tracings of the paddleto-breast contact regions and the outer breast borders on those transparencies. The tracings were drawn for 8 breasts both with and without using a rubber dam. The tracings were then digitized with a flatbed scanner and analyzed using the public domain computer program ImageJ (http://rsb.info.nih.gov/ij/docs/intro.html). The contact and outer breast contours were traced by hand in ImageJ, and the areas were calculated using the measure function. Also, the lengths of the gaps between the border of the contact 
region and the outer border of the breast in the tracings were measured at 5 different angles $\left(30^{\circ}\right.$, $60^{\circ}, 90^{\circ}, 120^{\circ}$, and $150^{\circ}$ ).

For both the "with" and "without" dam cases, after the contours were traced, the transparencies were removed, and ultrasound gel was distributed over the breast using a syringe. The ultrasound translation system was lowered to the compression paddle so the transducer was in contact with the gel/breast, and 3D ultrasound images were acquired. Two readers independently reviewed the 3D ultrasound images in a blinded study. The images were displayed using the ImageJ program. The readers were asked to rate the overall breast image quality, especially the breast coverage and image artifacts, on a scale of 1 through 5, with 1 being "much better image quality without the rubber dam," 3 being "equivalent image quality," and 5 being "much better image quality with the rubber dam."

Figure 3. Visual tracings of regions of the breast in contact with the paddle (inner curves) and outer breast borders in the same breast. A, Without a rubber dam, the percentage of the breast area in contact with the paddle is smaller, about $55.2 \%$. B, With a rubber dam and slight compression force, the percentage of the breast area in contact with the paddle is larger, approximately $70 \%$.

\section{A}

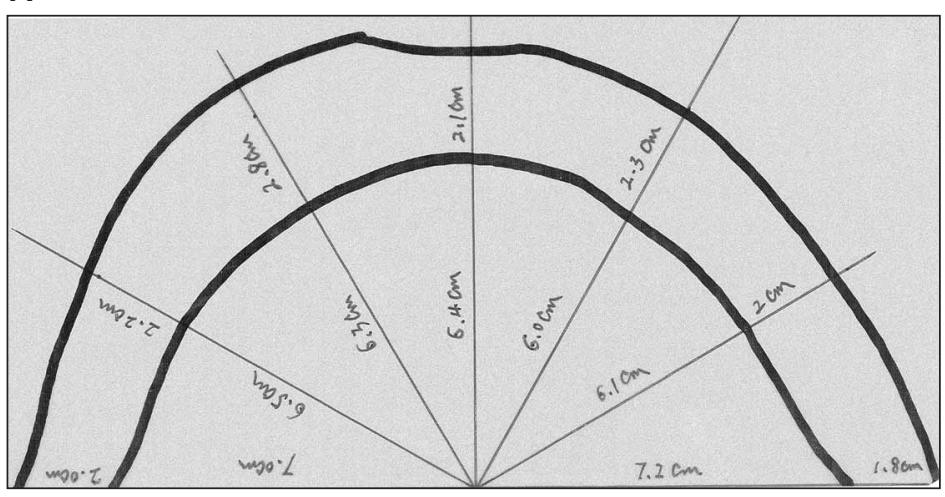

B

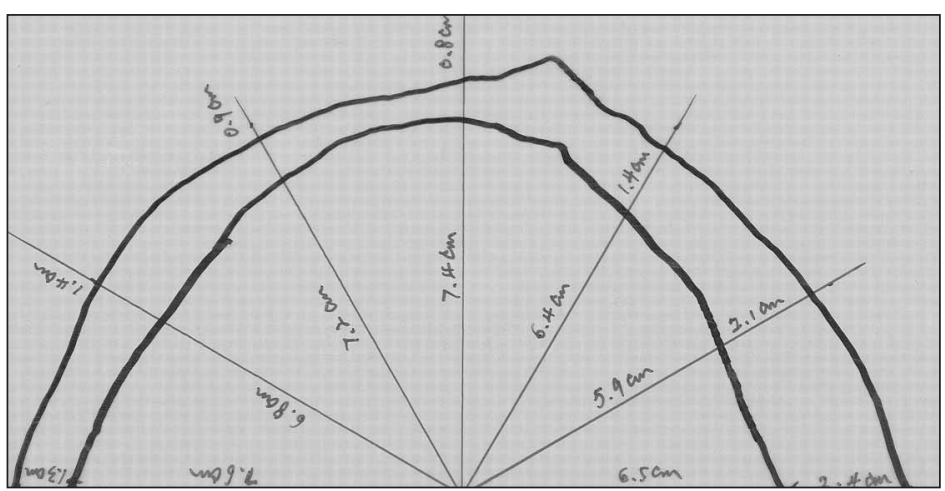

Statistical analyses were performed with Excel (Microsoft Corporation, Redmond, WA). Breast coverage-related parameters were calculated and reported as mean \pm SD. The coverage-related parameters derived from the scans using a rubber dam and those derived from the scans not using a dam were compared by a paired 2-tailed Student $t$ test. The level of significance was set to $P<.05$.

\section{Results}

Examples of the visual traces of the breastpaddle contact areas and breast outer boundaries for the with and without dam cases are shown in Figure 3.

The linear dimensions of the gaps between the border of the contact region and the outer border of the breast in the tracings for the with and without dam cases are listed in Table 1. For the 8 breasts in this study, the linear dimensions of the breast in contact with the paddle were larger; the noncontact gaps at the breast periphery were smaller; and the percentages of the breast linear dimensions in contact with the paddle were larger with the rubber dam than those without using the dam at all 5 different angles. Furthermore, the differences in noncontact gaps at the breast periphery and percentages of breast in contact with the paddle between using a rubber dam and not using it were all statistically significant $(P<.05)$.

The percentages of the breast areas in contact with the compression paddle from the visual tracings are listed in Table 2. There were significant differences in the breast areas in contact with the paddle and the total breast areas between using a rubber dam and not using it $(P<.05 ; P<.01$, respectively). The breast areas in contact with the paddle were larger $(69.9 \pm 46.7$ versus $61.2 \pm 42.3 \mathrm{~cm}^{2}$ ), and total breast areas were smaller $\left(88.5 \pm 48.5\right.$ versus $\left.99.1 \pm 49.1 \mathrm{~cm}^{2}\right)$, with the rubber dam than without it. A significant difference was also found in the percentages of the breast areas in contact with the paddle between using the rubber dam and not using it $(P<.01)$. The percentages of the breast areas in contact with the paddle were approximately $75.9 \% \pm 8.8 \%$ (range, $64.3 \%-90.4 \%$ ) with rubber dam use and $59.0 \% \pm 9.2 \%$ (range, $48.3 \%-79.5 \%$ ) without it. 
Table 1. Linear Dimensions of the Gaps at the Breast Periphery From Visual Tracings

\begin{tabular}{|c|c|c|c|c|c|c|c|c|c|}
\hline \multirow[b]{2}{*}{ Angle, ${ }^{\circ}$} & \multicolumn{3}{|c|}{$\begin{array}{l}\text { Breast in Contact } \\
\text { With Paddle, cm }\end{array}$} & \multicolumn{3}{|c|}{$\begin{array}{l}\text { Noncontact Gaps at the } \\
\text { Breast Periphery, cm }\end{array}$} & \multicolumn{3}{|c|}{$\begin{array}{l}\text { Breast in Contact } \\
\text { With Paddle, \% }\end{array}$} \\
\hline & Without Dam & With Dam & $P$ & Without Dam & With Dam & $P$ & Without Dam & With Dam & $P$ \\
\hline 30 & $6.19 \pm 1.86$ & $6.54 \pm 2.16$ & .14 & $1.76 \pm 0.36$ & $1.00 \pm 0.67$ & .01 & $75.9 \pm 5.7$ & $85.8 \pm 9.9$ & .01 \\
\hline 60 & $5.55 \pm 2.12$ & $5.80 \pm 2.16$ & .21 & $1.76 \pm 0.39$ & $0.96 \pm 0.36$ & .001 & $74.5 \pm 7.4$ & $84.3 \pm 8.0$ & .002 \\
\hline 90 & $5.30 \pm 2.06$ & $5.65 \pm 2.17$ & .08 & $1.75 \pm 0.43$ & $0.96 \pm 0.32$ & .006 & $74.3 \pm 4.5$ & $84.2 \pm 6.3$ & .008 \\
\hline 120 & $5.33 \pm 2.07$ & $5.79 \pm 2.38$ & .04 & $1.81 \pm 0.55$ & $0.91 \pm 0.40$ & .008 & $73.6 \pm 6.2$ & $84.5 \pm 9.0$ & .01 \\
\hline 150 & $5.91 \pm 1.99$ & $6.46 \pm 1.88$ & .08 & $1.83 \pm 0.66$ & $1.00 \pm 0.52$ & .01 & $75.4 \pm 9.2$ & $86.0 \pm 7.0$ & .01 \\
\hline
\end{tabular}

Values are mean \pm SD.

With the dam, the mean estimated area of the breast in contact with the paddle increased $14.4 \% \pm 14.3 \%$, whereas the mean increase in the fraction of the total breast area in contact with paddle was $30.2 \% \pm 18.7 \%$. The difference was due to the mean total projected area of the breast decreasing $11.7 \% \pm 7.6 \%$ as the dam was pressed against it.

Figure 4 compares the relative image quality obtained with the ABU scanning system using a rubber dam surrounding the breast periphery and without using it. The rubber dam substantially improved breast coverage because it reduced gel slippage away from the breast periphery.

The image quality of ABU using a rubber dam was consistently judged to be superior to that without using it, as the readers expressed a mean preference of 4.3 on the scale of 1 through 5 described above. Figure 5 shows the 2 readers' ratings of the ultrasound image quality.

\section{Discussion}

Ultrasound has been a valuable supplement to mammography in characterizing and even detecting breast lesions in high-risk women, including particularly those with mammographically dense breasts. ${ }^{2,413,14}$ Automated ultrasound in our current implementation in the mammographic geometry probably cannot yet entirely replace clinical hand ultrasound scans for screening purposes because of the presence of air bubbles in the coupling medium, the lack of full breast volume coverage, possibly less than optimal acquisition due to the practical limitation of using only 1 or 2 (eg, craniocaudal and mediolateral oblique) views of tissues of interest in the 3D scans, and the reduced compression when the whole breast is compressed. However, less interpretation is required at the time of scanning, and anatomic correlation of ultrasound with mammography or tomosynthesis aids the radiologist's evaluation of a suspicious mass in the human breast and raises the confidence level of his or her assessment.

As discussed in a previous study, ${ }^{8}$ if gel does not liberally fill the gaps between the breast and the compression paddle at the breast periphery, ultrasound coverage can be limited to only half of the total breast area for the combined $\mathrm{x}$ ray/ultrasound imaging system. Coverage of the periphery can be critical because $73 \%$ of malig-

Table 2. Percentage of Breast Area in Contact With the Compression Paddle From Visual Tracings

\begin{tabular}{|c|c|c|c|c|c|c|c|}
\hline \multirow[b]{2}{*}{ Breast } & \multicolumn{2}{|c|}{$\begin{array}{c}\text { Area in Contact } \\
\text { With Paddle, } \mathrm{cm}^{2}\end{array}$} & \multicolumn{2}{|c|}{$\begin{array}{l}\text { Total Breast } \\
\text { Area, } \mathrm{cm}^{2}\end{array}$} & \multicolumn{2}{|c|}{$\begin{array}{c}\text { Breast Area in Contact } \\
\text { With Paddle, \% }\end{array}$} & \multirow{2}{*}{$\begin{array}{l}\text { Increase in Fraction } \\
\text { of Breast Area, } \%\end{array}$} \\
\hline & Without Dam & With Dam & Without Dam & With Dam & Without Dam & With Dam & \\
\hline 1 & 63.49 & 74.31 & 115.07 & 106.18 & 55.2 & 70 & 26.8 \\
\hline 2 & 52.67 & 64.89 & 109.01 & 82.17 & 48.3 & 79 & 63.6 \\
\hline 3 & 66.03 & 81.18 & 110.19 & 94.5 & 59.9 & 85.9 & 43.4 \\
\hline 4 & 159.23 & 174.53 & 200.25 & 193 & 79.5 & 90.4 & 13.7 \\
\hline 5 & 49.61 & 62.22 & 85.62 & 83.95 & 57.9 & 74.1 & 28 \\
\hline 6 & 46.39 & 46.85 & 81.13 & 68.29 & 57.2 & 68.6 & 19.9 \\
\hline 7 & 22.79 & 29.42 & 43.16 & 39.38 & 52.8 & 74.7 & 41.5 \\
\hline 8 & 29.68 & 25.88 & 48.52 & 40.26 & 61.2 & 64.3 & 5.1 \\
\hline
\end{tabular}


nant lesions in breasts of women younger than 50 years have been found at the periphery of the breast, as defined by a zone $1 \mathrm{~cm}$ wide within the subcutaneous fat or anterior to the retromammary fat. ${ }^{15}$

Figure 4. A, Single slice of a gray scale image volume without a rubber dam around the breast border. $\mathbf{B}$, Same image slice using a rubber dam surrounding the breast periphery. The rubber dam substantially improved breast coverage. The arrow indicates the improved area of coverage because of using the rubber dam.

A

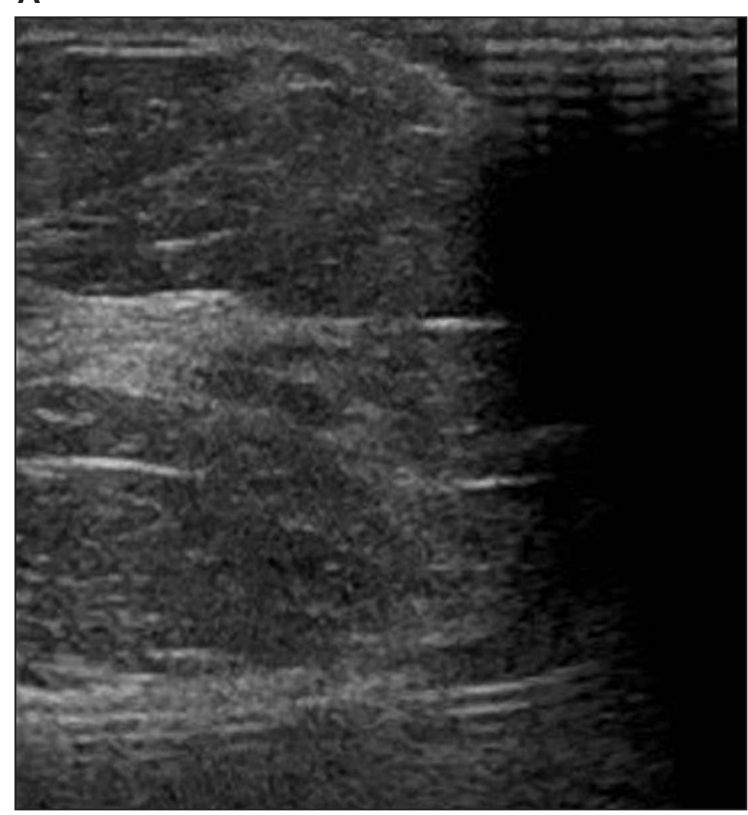

B

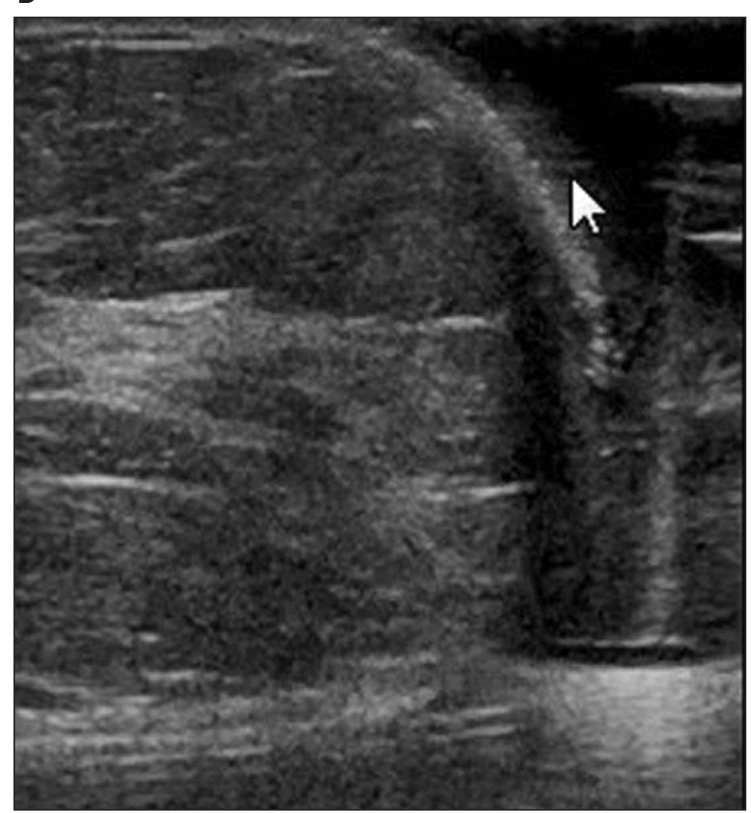

Use of the here-introduced rubber dam enhanced the percentage of the breast (tissue) area in contact with the paddle by $30 \%$ without changing the compressed breast thickness. Use of the dam resisted gel slippage and (probably) reduced reverberations in the gel by absorbing some of the extraneous ultrasound. Retained gel at the periphery of the breast allowed better imaging of the subareolar region. The overall image quality of $A B U$ using the introduced rubber dam was consistently judged superior to that without using it. The gel could be easily dispensed to the breast periphery when using the rubber dam with (probable) reduction in air bubbles as well. The variable angle (shape) capability and height differences of the rubber dams accommodate different breast sizes. The dam can be easily pushed toward the chest wall, and as long as the pushing against the breast is not too forceful, posterior movement of breast tissues outside the ultrasound scanning range is not likely, and spatial correlation between structures in $\mathrm{ABU}$ and DBT, determined before deployment of the dam, are not affected greatly.

Finally, it should be noted that in our study, artifact reflections were sometimes observed in the ABU images with the dams. These reflections arise because of the grazing incidence of the ultrasonic waves on the flat rubber surface of the dam. The reflections could be reduced by using a sawtooth or other rough pattern on the rubber surface. Also in this study, some variability in measurements was caused by the protective

Figure 5. Ultrasound image quality ratings for 8 cases by 2 readers on the scale of 1 through 5 described in "Materials and Methods."

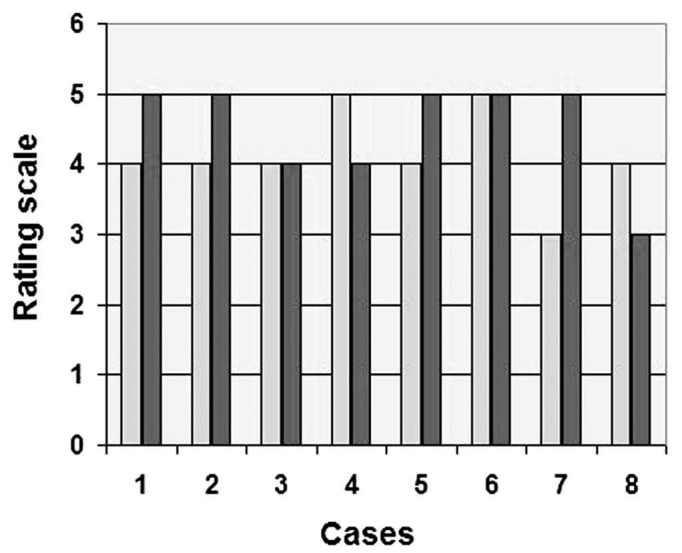


cowling for the tomosynthesis, although particular care was taken to reposition the same breast consistently. We are in the process of replacing the current cowling with a more ergonomic one.

In summary, the rubber dam method in $\mathrm{ABU}$ in the mammographic geometry increases breast coverage by shaping the breast and retaining gel at the periphery to reduce noncontact gaps. The image quality of $\mathrm{ABU}$ using the rubber dam was superior to that without its use.

\section{References}

1. Jackson VP. The role of US in breast imaging. Radiology 1990; 177:305-311.

2. Taylor KJ, Merritt C, Piccoli C, et al. Ultrasound as a complement to mammography and breast examination to characterize breast masses. Ultrasound Med Biol 2002; 28:19-26.

3. Conway WF, Hayes CW, Brewer WH. Occult breast masses: use of a mammographic localizing grid for US evaluation. Radiology 1991; 181:143-146.

4. Kolb TM, Lichy J, Newhouse JH. Occult cancer in women with dense breasts: detection with screening US - diagnostic yield and tumor characteristics. Radiology 1998; 207:191-199.

5. Berg WA. Rationale for a trial of screening breast ultrasound: American College of Radiology Imaging Network (ACRIN) 6666. AJR Am J Roentgenol 2003; 180:12251228

6. Kapur A, Carson PL, Eberhard J, et al. Combination of digital mammography with semi-automated 3D breast ultrasound. Technol Cancer Res Treat 2004; 3:325-334.

7. Booi RC, Krücker JF, Goodsitt MM, et al. Evaluating thin compression paddles for mammographically compatible ultrasound. Ultrasound Med Biol 2007; 33:472-482.

8. Sinha SP, Goodsitt MM, Roubidoux MA, et al. Automated ultrasound scanning on a dual-modality breast imaging system: coverage and motion issues and solutions. J Ultrasound Med 2007; 26:645-655.

9. Sinha SP, Roubidou MA, Helvie MA, et al. Multi-modality 3D breast imaging with $X$-ray tomosynthesis and automated ultrasound. Conf Proc IEEE Eng Med Biol Soc 2007; 2007:1335-1338.

10. Kotsianos-Hermle D, Wirth S, Fischer T, Hiltawsky KM, Reiser M. First clinical use of a standardized three-dimensional ultrasound for breast Imaging. Eur J Radiol 2009; 71:102-108.

11. Shipley JA, Duck FA, Goddard DA, et al. Automated quantitative volumetric breast ultrasound data-acquisition system. Ultrasound Med Biol 2005; 31:905-917.

12. Blane CE, Goodsitt MM, Grimm JC, et al. New compression paddle for wire localization in mammography. Acad Radiol 2010; 17:142-145.
13. Moss HA, Britton PD, Flower CDR, Freeman AH, Lomas DJ, Warren RM. How reliable is modern breast imaging in differentiating benign from malignant breast lesions in the symptomatic population? Clin Radiol 1999; 54:676-682.

14. Berg WA, Blume JD, Cormack JB, et al. Combined screening with ultrasound and mammography vs mammography alone in women at elevated risk of breast cancer. JAMA 2008; 299:2151-2163.

15. Stacey-Clear A, McCarthy KA, Hall DA, et al. Mammographically detected breast cancer: location in women under 50 years old. Radiology 1993; 186:677680. 